\title{
Acute Care Utilization by Dementia Caregivers Within Urban Primary Care Practices
}

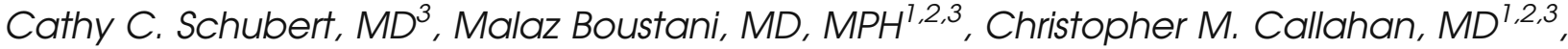 \\ Anthony J. Perkins, $\mathrm{MS}^{1,2}$, Siu Hui, PhD ${ }^{1,2,3}$, and Hugh C. Hendrie, MB, ChB $B^{1,2,4}$ \\ ${ }^{1}$ Indiana University Center for Aging Research, Indianapolis, IN, USA; ${ }^{2}$ Regenstrief Institute, Inc., Indianapolis, IN, USA; ${ }^{3}$ Department of \\ Medicine, Indiana University School of Medicine, Indianapolis, IN, USA; ${ }^{4}$ Department of Psychiatry, Indiana University School of Medicine, \\ Indianapolis, IN, USA.
}

BACKGROUND: Caring for an individual with Alzheimer's dementia $(\mathrm{AD})$ is stressful, and studies show that this stress has an impact on both the physical and mental health of the caregiver. However, many questions remain about the characteristics of $\mathrm{AD}$ patients and their caregivers that contribute to this stress and how it impacts caregivers' use of healthcare resources.

OBJECTIVE: To study the impact of stress on the physical and mental health of the caregiver.

DESIGN: Patients underwent extensive testing to allow description of their degree of cognitive impairment, behavioral and psychological symptoms, medical comorbidities, and functional abilities. Caregivers were assessed for depressive symptoms and also for emergency department (ED) use and hospitalizations in the previous six months. Multivariate logistic regression was used to evaluate impact of patients' dementia symptoms on caregivers' acute care utilization.

PARTICIPANTS: One hundred and fifty-three $\mathrm{AD}$ patients and their caregivers attending two large, urban, university-affiliated primary care practices were enrolled in a cross-sectional study to examine the facets of dementia caregiving that impact caregiver acute health care utilization.

RESULTS: Twenty-four percent of the caregivers had at least one ED visit or hospitalization in the six months prior to enrollment. After adjusting for caregiver age, gender, and education, our logistic regression model found that the caregivers' acute care utilization was associated with their depression as measured by the PHQ-9 (OR 1.09, 95\% CI 1.00-1.18), the patients' behavioral and psychological symptoms as measured by the NPI (OR 1.04, 95\% CI 1.01-1.08), and the patients' functional status as measured by the ADCSADL (OR 1.05, 95\% CI 1.01-1.09).

CONCLUSION: To improve the health of $\mathrm{AD}$ caregivers, a primary care system needs to reallocate resources to manage the functional, behavioral, and psychological symptoms related to the care-recipients suffering from $\mathrm{AD}$.

Received July 18, 2007

Revised February 5, 2008

Accepted June 17, 2008

Published online August 9, 2008
KEY WORDS: health care utilization; ED visit; hospitalization; dementia; caregiver.

J Gen Intern Med 23(11):1736-40

DOI: $10.1007 / \mathrm{s} 11606-008-0711-0$

(c) Society of General Internal Medicine 2008

\section{INTRODUCTION}

Approximately four million older adults in the United States have Alzheimer's dementia (AD), and three million of them are living in the community. ${ }^{1}$ Family and friends are currently providing $75 \%$ of the daily care needs of these patients, with the remaining $25 \%$ being provided by purchased home care services. ${ }^{2}$ Thus, dementing illnesses are complex in that they impact not only the health and function of the patient but also that of the caregiving family member or friend. With the aging of the population, the number of cases of $\mathrm{AD}$ is anticipated to increase to 18.5 million by $2050 .^{3}$ If a similar proportion of $\mathrm{AD}$ patients remains in the community as now, the burden of caregiving on the family and friends by 2050 will increase exponentially.

Caregiving is a stressful endeavor, and caring for a patient with a dementing illness appears to be even more so. In a national survey of more than 1,500 family caregivers, the caregivers of demented patients described their duties as more stressful compared to how caregivers of physically-impaired but not demented older adults viewed theirs. The dementia caregivers also reported spending significantly more hours per week providing care, giving up their vacations or hobbies more often, having less time for other family members, and having more work-related problems. ${ }^{4}$

To evaluate the psychological effects of this stress of caregiving, Schulz et al. reviewed the dementia literature to evaluate the prevalence and magnitude of psychiatric complaints in caregivers. ${ }^{5}$ Almost all studies reported increased levels of depressive symptoms in caregivers, and this psychiatric morbidity was found to be correlated with not only patient problem behaviors but also caregiver income, self-rated health, perceived stress, and life satisfaction. In a more recent review, Cuijpers systematically examined the dementia caregiving literature for studies reporting actual diagnosis of a major depressive disorder. ${ }^{6}$ He found that incidence and prevalence of full-blown depression are also increased in caregivers of dementia patients when compared to the non-caregiving population. Thus, we know that the stress involved in caring 
for someone with dementia impacts the caregivers' mental health.

Studies have also examined whether dementia caregiving has an effect on the caregivers' physical health. Spousal caregivers of patients with $\mathrm{AD}$ appear to have a greater risk for developing serious illness, ${ }^{7}$ and being a caregiver under mental or emotional strain was found to be an independent risk factor for mortality in older spousal caregivers. ${ }^{8}$ Vitaliano et al. conducted a meta-analysis of the dementia caregiving literature. ${ }^{9}$ In the analysis, they combined the results of 23 studies that compared indicators of physical health in family caregivers of demented patients to health indicators in noncaregiver age- and sex-matched controls. Overall, caregivers were found to have higher amounts of circulating stress hormones and lower degrees of antibody responses compared to the non-caregivers, but a definitive conclusion of whether caregiving is directly hazardous to health could not be determined.

Primary care physicians provide most of the medical care both for patients with $\mathrm{AD}$ and for their caregivers. With the aging of the population and the subsequent increase in patients with $\mathrm{AD}$, the effects of caregiving on the physical and mental health of caregivers will continue to be important at both the individual and societal level. A better understanding of what makes caregiving of the demented more stressful is needed, as is more study into how medical comorbidity of the patient and the caregiver impacts their respective clinical pictures. Where care of patients with $\mathrm{AD}$ is concerned, such study may indicate that primary care providers need to expand their definition of "patient" to include not only the demented individual but the caregiver as well.

We describe a clinical profile of the medical comorbidity and the cognitive, functional, behavioral and psychological symptoms (BPSD) of a cohort of patients with $\mathrm{AD}$. We then use this profile to examine the impact of these symptoms on their caregivers' emergency department and hospital utilizations. Based on our clinical experience and previous research, our primary hypothesis is that $\mathrm{AD}$ patients' $\mathrm{BPSD}$, not their cognition, will be associated with their caregivers' acute care utilization.

\section{METHODS}

The study was approved by the Indiana University Purdue University-Indianapolis Institutional Review Board. All subjects or their caregivers provided written informed consent for participation.

Patients with $\mathrm{AD}$ were recruited from two large primary care practices from January 2002 to August 2004. The first practice included seven community-based health centers affiliated with Wishard Health Services, a university-affiliated urban health care system serving medically indigent patients in Indianapolis. This practice serves approximately 5,000 older adults. The second site included three primary care practices at the Roudebush Veterans Affairs Medical Center in Indianapolis. This practice provides primary care to approximately 6,000 veterans age 65 and older.

Patients were recruited through two methods: (1) physician referral following a written prompt that the patient may be eligible due to a medical record diagnosis consistent with a dementing illness; and (2) physician referral following a written prompt from the research team that the patient screened positive on cognitive testing. All patients aged 65 and older who were receiving primary care from one of the two sites were screened for dementia using a six-item screener instrument ${ }^{10}$ and an abbreviated version of the Community Screening Instrument for Dementia (CSI-D). ${ }^{11}$ All referred patients completed a formal diagnostic evaluation that included neuropsychological testing, comprehensive physical examination, a structured interview with the caregiver, brain imaging, and reversible work-up for dementia. All results were reviewed by a consensus diagnosis panel including a psychologist, neuropsychologist, geriatrician, and a geriatric psychiatrist. Subjects were eligible if they met criteria for possible or probable $\mathrm{AD}$ based on ICD-10 criteria. This diagnostic method is described in more detail elsewhere. ${ }^{12}$ Exclusion criteria included: residence in a nursing home, unable to understand English, no access to a telephone, or no caregiver willing to consent to participate in the study. The primary care physician could then refer the patient to be enrolled in a randomized trial to test the effectiveness of collaborative care management for older adults with $\mathrm{AD}$ compared with augmented usual care. In this study, caregivers were family members or friends who identified themselves as individuals who assisted the subjects with navigating daily life and who were willing to participate in the study.

Patient Data. Following enrollment into the clinical trial and as part of the baseline assessment, the caregivers completed an assessment by telephone and thus were the primary source of the patient's data. The baseline interview included standardized instruments developed by the Consortium of Alzheimer Disease Center investigators ${ }^{13}$ and are considered the current gold standard for health outcome measures for patients with $\mathrm{AD}$ and their caregivers. These included the Neuropsychiatric Inventory (NPI), ${ }^{14}$ the Alzheimer's Disease Cooperative Study-Activities of Daily Living Inventory (ADCSADL), ${ }^{15}$ and Resource Use Inventory (RUI). ${ }^{13,16}$ The NPI has been adopted by the Alzheimer's Disease Cooperative Studies (ADCS) Group to obtain information on the presence of psychopathology in ten behavioral areas, including delusions, apathy, hallucinations, disinhibition, agitation, depression, aberrant motor behavior, anxiety, night-time behavior, and euphoria. Possible scores range from 0-120, with high numbers indicating more behavioral problems. The test has excellent reliability and validity. ${ }^{14,17,18}$ The ADCS-ADL is a 23-item inventory developed by the ADCS Group and is administered to the patient's caregiver by a trained interviewer. The caregiver is asked to focus on the patient's performance over the past month. Notably, the caregiver reports on what the patient actually did rather than an assessment of what the patient might be able to do. Thus, the inventory focuses on observed actions. The instrument assesses the traditional basic activities of daily living as well as variations on instrumental activities of daily living and a number of more complex and explicit self-care tasks. ${ }^{15}$ Patients' scores range from 0-75, with higher scores indicating greater levels of function. The RUI is a 15-item questionnaire developed by the ADCS Group that is designed to measure the health care utilization and costs incurred by subjects with $\mathrm{AD}$. Three categories of costs and use are assessed, including medical services, discretionary health care costs, and caregiver expenses. It inquires specifically about nursing home stays 
as well as home health visits, clinic visits, and hospitalizations. Even though studies have proven that the RUI provides data that are reliable and valid, ${ }^{16}$ in our previous studies we found that older adults have difficulty recalling use over the prior year. ${ }^{19}$ Thus, we completed the RUI for the previous six months to improve recall.

All patients themselves completed the Telephone Interview for Cognitive Status (TICS), a telephone version of the MiniMental State Examination ${ }^{20}$ that has the same score range from 0 to 30 with the higher number indicating less cognitive impairment.

Using each patient's list of prescribed medications, we calculated the Chronic Disease Score (CDS) as a measure of medical comorbidity. ${ }^{21}$ The CDS excludes medications used for treatment of acute problems (such as antibiotics) or common symptoms (such as nasal congestion). Individual medications are assigned to pharmacy classes, which are then mapped to the chronic diseases that class of medication would treat. Each CDS class was assigned a weight by the original developers based on expert judgment. These weights are used to calculate the patient's total CDS (range 0-24). The CDS has been validated as an indicator of comorbidity, and its scores are correlated with future resource utilization. Higher scores indicate greater chronic disease burden and risk of healthcare resource utilization.

Caregiver Data. In addition to providing information about the patient's status, the caregiver provided information about their own demographics, their mood (PHQ-9), and their acute care utilization (RUI). The Patient Health Questionnaire-9 (PHQ-9) ${ }^{22}$ assesses incidence and severity of each of the nine DSM-IV criteria for depression, including anhedonia, appetite changes, low energy, and difficulty concentrating. Scores can range from 0-27, with higher scores indicating worsening depression. They also completed the RUI as a measure of their own acute healthcare utilization.

\section{ANALYSIS}

We used two sample T-tests and Fisher's exact test to compare the demographic, neuropsychiatric inventory, and survey measures of caregivers with an ED visit or hospital admission to those without. We then used multivariate logistic regression models to evaluate the impact of patients' overall medical comorbidity and dementia symptoms (functional, cognitive, behavioral, and psychological symptoms) on the caregiver acute utilization. Caregiver age, gender, education, and depression (PHQ-9 score) were specified for model inclusion regardless of $\mathrm{p}$-value due to their clinical relevance and importance.

\section{RESULTS}

Our study included 153 patients meeting ICD-10 criteria for $\mathrm{AD}$ and their caregivers (Table 1). The patients' mean age was 77.7 years (SD 5.8), 43\% were female, and 49\% were African American. The average TICS score was 18.1 (SD 5.6) (indicating mild to moderate dementia), the mean ADCS-ADL scale score was 50.0 (SD 15.8) (indicating fair patient functional level), and the average NPI-12 score was 11.8 (SD 18.2) (indicating moderate levels of BPSD). Caregivers had an average age that was 16 years younger than the patients, $69 \%$ of them lived with the patient, and $89 \%$ were female.

Twenty-four percent of caregivers had at least one ED visit or hospitalization. In assessing for caregiver characteristics associated with this acute care utilization, bivariate analyses found that these caregivers were caring for patients with more BPSD (mean NPI 20.5 vs. 9, p=0.023) and less cognitive impairment (mean TICS 19.1 vs. 16.6, p=0.057) and that these caregivers suffered from more depressive symptoms themselves (mean PHQ 6.1 vs. 3.4, p=0.044) (see Table 2).

Using multivariate logistic regression analyses to adjust for the caregivers' age, gender, education, and level of depression, we found that caregiver acute care utilization was associated with the patients' behavioral and psychological symptoms $(\mathrm{OR}=1.04$, 95\% CI 1.01-1.08 for each one point increase on the NPI) and their functional status (OR=1.05, 95\% CI 1.01-1.09 for each one point increase on the ADCS-ADL). However, we found no association between caregivers' acute care utilization and the patients' comorbidity or their cognitive function (see Table 3).

\section{DISCUSSION}

Our cross-sectional study conducted among AD patients and their caregivers found that patients' function and BPSD were associated with utilization of acute care services by their caregivers. We found no association between patients' level of cognitive impairment and caregivers' acute care utilization. Interestingly, this is somewhat different from what Shaw et al. found in their study focusing on spousal caregivers of $\mathrm{AD}$ patients. As in our study, when compared to controls, spousal caregivers in Shaw et al.'s study showed a trend of higher risk for having a serious illness that was associated with providing

Table 1. Characteristics of Patients and Caregivers

\begin{tabular}{ll}
\hline \hline & $\mathrm{N}=153$ \\
\hline Patient variables & $\%$ or Mean (SD) \\
\% Female & 43.1 \\
\% African American & 49.0 \\
Mean age & $77.7(5.8)$ \\
Mean years of education & $9.2(4.2)$ \\
\% Married & 48.4 \\
Mean TICS & $18.1(5.6)$ \\
Mean ADCS-ADL & $50.0(15.8)$ \\
Mean NPI & $11.8(18.2)$ \\
Mean CDS & $7.8(3.9)$ \\
Caregiver variables & \\
\% Female & 88.9 \\
Mean age & $60.9(15.0)$ \\
Mean years of education & $11.9(2.7)$ \\
\% Live with patient & 69.3 \\
\% Patient's spouse & 44.4 \\
Mean PHQ-9 & $4.1(5.3)$ \\
\% ED visit prior 6 months & 20.9 \\
\% EDpatient admission prior 6 months & 10.5 \\
\hline
\end{tabular}

* TICS $=$ Telephone Interview for Cognitive Status, ADCS-ADL = Alzheimer's Disease Cooperative Study-Activities of Daily Living, NPI = Neuropsychiatric Inventory, CDS = Chronic Disease Score, ED = emergency department, PHQ-9 = Patient Health Questionnaire 
Table 2. Comparison of Caregivers by ED Visit or Inpatient Admission

\begin{tabular}{|c|c|c|c|}
\hline & \multicolumn{2}{|c|}{$\begin{array}{l}\text { ED visit or inpatient } \\
\text { admission by caregiver }\end{array}$} & \multirow[t]{2}{*}{ P-value } \\
\hline & None $\mathrm{N}=116$ & Any $\mathrm{N}=37$ & \\
\hline Mean age: patient & $78.0(5.6)$ & 76.7 (6.3) & 0.271 \\
\hline $\begin{array}{l}\text { Mean years of education: } \\
\text { patient }\end{array}$ & $9.3(4.4)$ & $8.6(3.7)$ & 0.362 \\
\hline $\begin{array}{l}\text { Mean ADCS-ADL score: } \\
\text { patient }\end{array}$ & $49.4(16.3)$ & $52.0(14.0)$ & 0.352 \\
\hline Mean CDS: patient & $7.9(4.1)$ & $7.4(3.5)$ & 0.464 \\
\hline Mean TICS: patient & $17.2(8.1)$ & $18.6(6.2)$ & 0.293 \\
\hline Mean NPI: patient & $9.0(12.1)$ & $20.5(28.7)$ & 0.023 \\
\hline \% Female: patient & 20.7 & 28.8 & 0.259 \\
\hline Mean age: caregiver & $61.8(14.7)$ & $58.0(15.5)$ & 0.199 \\
\hline $\begin{array}{l}\text { Mean years of education: } \\
\text { caregiver }\end{array}$ & $12.1(2.6)$ & $11.5(2.8)$ & 0.264 \\
\hline Mean PHQ-9: caregiver & $3.4(4.2)$ & $6.1(7.6)$ & 0.044 \\
\hline \% Female: caregiver & 29.4 & 23.5 & 0.595 \\
\hline
\end{tabular}

* ADCS-ADL $=$ Alzheimer's Disease Cooperative Study-Activities of Daily Living, $\mathrm{CDS}=$ Chronic Disease Score, TICS $=$ Telephone Interview for Cognitive Status, NPI = Neuropsychiatric Inventory, PHQ-9 = Patient Health Questionnaire

more ADL and functional assistance to the patient; however, they found no association with the patients' BPSD. ${ }^{7}$ As Shaw's study focused exclusively on spousal caregivers and ours, however, included any informal caregiver, direct comparisons cannot be made.

This association between patients' function and BPSD and caregiver use of health care services is an indicator of the difficulty of managing and caring for patients suffering from $\mathrm{AD}$. In fact, BPSD, but not cognitive impairment, have been shown to be a prominent factor in the caregiver's decision to seek long-term residential placement for the patient. ${ }^{23}$ Thus, it is not as much the patient's loss of cognitive function that stresses caregivers, perhaps because the caregiver expects and is prepared for this as part of the dementing illness. Rather, the agitation, aggression, and other symptoms contribute most heavily to caregiver stress and burden.

As our population ages and the numbers of $\mathrm{AD}$ cases increases, by necessity the number of caregivers, both formal and informal, will also increase. Unless our medical system can begin better to address and treat the BPSD of the demented patient, caregiver stress will continue unabated. In addition, our study found that the caregivers with more depressive symptoms were more likely to utilize acute health-

Table 3. Logistic Regression Model of ED Visit or Inpatient Admission by Caregivers

\begin{tabular}{llll}
\hline \hline & Odds ratio & $\mathbf{9 5 \% ~ C l}$ & P-value \\
\hline Caregiver age & 0.99 & $(0.97,1.03)$ & 0.974 \\
Female caregiver & 0.47 & $(0.13,1.64)$ & 0.235 \\
Caregiver education & 0.88 & $(0.74,1.05)$ & 0.153 \\
Caregiver PHQ-9 & 1.09 & $(1.00,1.18)$ & 0.048 \\
NPI & 1.04 & $(1.01,1.08)$ & 0.014 \\
ADCS-ADL & 1.05 & $(1.01 .1 .09)$ & 0.023 \\
TICS & 1.02 & $(0.96,1.09)$ & 0.540 \\
CDS & 0.98 & $(0.88,1.10)$ & 0.780 \\
\hline
\end{tabular}

PHQ-9 = Patient Health Questionnaire, NPI = Neuropsychiatric Inventory, ADCS-ADL = Alzheimer's Disease Cooperative Study-Activities of Daily Living, TICS = Telephone Interview for Cognitive Status, CDS = Chronic Disease Score care resources. This is in line with the results of other studies of depression and its impact on use of health resources. ${ }^{24}$ Thus, to truly impact the care of the $\mathrm{AD}$ patient and caregiver, we need to include assessment and treatment of psychological symptoms of the caregiver, too.

Our study demonstrated that $\mathrm{AD}$ caregiving produces significant stress that impacts both the medical and psychological wellbeing of caregivers. Thus, our primary health care system needs to change its approach to providing medical care to $\mathrm{AD}$ patients. Rather than caring only for the demented patient, primary care providers will need to expand their definition of "patient" to include the caregiver as well.

Collaborative care programs within the primary care and home settings offer the best possibility for managing patients' symptoms while supporting the caregivers' efforts to care for them at home and reducing the need for utilization of acute care services. ${ }^{25-27}$ Recent trials of such collaborative interventions included in-home education and psychosocial support for the caregiver on issues such as caregiver depression and burden and patients' BPSD. ${ }^{25-27}$ The collaborative interventions studied were successful in reducing caregiver stress and improving quality of life. ${ }^{25-27}$ In addition, patient's behavioral and psychological symptoms were reduced as a result of the collaborative interventions. ${ }^{25}$ However, further research is needed to fully describe such a program and to evaluate its feasibility in clinical practice.

Our study has important internal and external validity limitations. First, the external validity of the findings is limited because our data are from patient-caregiver dyads that were willing to pursue both an evaluation of cognitive impairment, enroll in a clinical trial, and receive care in two specific health care systems. Our study group had a high percentage of African Americans, so the results may not be generalizable to all populations. On the other hand, minorities tend to be underrepresented in many studies of $\mathrm{AD}$, so our report offers a unique glimpse into how $\mathrm{AD}$ impacts a minority population. Second, the cross-sectional design of our study limits its internal validity and its capability of determining a causeand-effect relationship. Third, our information on ED/hospital utilization over the six months prior to the study was obtained from caregiver interview using the RUI. Thus, it is conceivable that some acute care visits were not reported and that our prevalence rate is conservative. Fourth, we did not collect information on the specific reasons for the caregivers' ED visits or hospitalizations, so we cannot comment on whether these were ambulatory care sensitive conditions such that caregiving duties might have interfered with non-emergent treatment or if an episode of patient's BPSD led directly to a caregiver ED visit.

Our study reveals the importance and impact of functional and BPSD symptoms in Alzheimer dementia on caregivers' use of acute healthcare services. Improved management of such symptoms in the primary care system may lead to a significant decrease in the burden of $\mathrm{AD}$, not just for the patient and caregiver but also for the healthcare system at large. Further research efforts are necessary to better describe effective, practical methods to provide this management in primary care.

Acknowledgements: Supported by Grant (R01HS10884-01) from the Agency for Healthcare Research and Buality. Dr. Schubert was supported by Hartford Foundation Center for Excellence in Geriatric 
Medicine and Dr. Boustani was supported by Paul A. Beeson Career Development Award in Aging (K23 AG 26770-01).

Conflict(s) of Interest: There are no conflicts of interest for these authors.

Corresponding Author: Malaz Boustani, MD, MPH; Regenstrief Institute, Inc., 410 West Tenth Street, Suite 2000, Indianapolis, IN 46202-3012, USA (e-mail: mboustani@regenstrief.org).

\section{REFERENCES}

1. Boustani M, Peterson B, Hanson L, Harris R, Lohr K. Screening for dementia in primary care: a summary of the evidence for the U.S. Preventive Services Task Force. Ann Intern Med. 2003;138:927-37.

2. Schulz R, Martire LM. Family caregiving of persons with dementia: prevalence, health effects, and support strategies. Am J Geriatr Psychiatry. 2004; 12:240-9.

3. Sloan FA, et al. Effect of alzheimer disease on the cost of treating other diseases. Alzheimer Dis Assoc Disord. 2002;16:137-43.

4. Ory MG, Hoffman RR, Yee JL, Tennstedt S, Schulz R. Prevalence and impact of caregiving: a detailed comparison between dementia and nondementia caregivers. Gerontologist. 1999;39:177-85.

5. Schulz R, et al. Psychiatric and physical morbidity effects of dementia caregiving: prevalence, correlates, and causes. Gerontologist. 1995;35:771-91.

6. Cuijpers P. Depressive disorders in caregivers of dementia patients: a systematic review. Aging Ment Health. 2005;9:325-30.

7. Shaw WS, Patterson TL, Semple SJ, et al.. Longitudinal analysis of multiple indicators of health decline among spousal caregivers. Annals Behav Med. 1997;19:101-9.

8. Schulz R, Beach SR. Caregiving as a risk factor for mortality: the caregiver health effects study. JAMA. 1999;282:2215-9.

9. Vitaliano $\mathbf{P}$, et al. Is caregiving hazardous to one's physical health? A meta-analysis. Psychol Bull. 2003;129:946-72.

10. Callahan CM, Unverzagt FW, Hui SL, et al. Six-item screener to identify cognitive impairment among potential subjects for clinical research. Med Care. 2002;40:771-81.

11. Hall KS, Gao S, Emsley CL, et al. Community screening interview for dementia (CSI 'D'); performance in five disparate study sites. Int'l $\mathrm{J}$ Geriatr Psychiatry. 2000;15:521-31.

12. Boustani M, Callahan CM, Unverzagt FW, et al. Implementing a screening and diagnosis program for dementia in primary care. J Gen Intern Med. 2005;20:572-7.
13. Ferris SH, Mackell JA, Mohs R, et al. A multicenter evaluation of new treatment efficacy instruments for Alzheimer's disease clinical trials: overview and general results. The Alzheimer's Disease Cooperative Study. Alzheimer Dis Assoc Disord. 1997;11(Suppl 2):S1-12.

14. Cummings JL, Mega M, Gray K, Rosenberg-Thompson S, Carusi DA Gornbein $\mathbf{J}$. The neuropsychiatric inventory: comprehensive assess ment of psychopathology in dementia. Neurology. 1994;44(12):230814. Dec.

15. Galasko D, Bennett D, Sano M, et al. An inventory to assess activities of daily living for clinical trials in Alzheimer's disease. The Alzheimer's Disease Cooperative Study. Alzheimer Dis Assoc Disord. 1997;11(Suppl 2):S33-9.

16. Sano $\mathbf{M}, \mathbf{Z h u} \mathbf{C W}$, Whitehouse PJ, et al. ADCS Prevention Instrument Project: Pharmacoeconomics: assessing health-related resource use among healthy elderly. Alzheimer Dis Assoc Disord. 2006;20(Suppl 3): S191-202

17. Kaufer DI, Cummings JL, Christine D, et al. Assessing the impact of neuropsychiatric symptoms in Alzheimer's disease: the neuropsychiatric inventory caregiver distress scale. J Am Geriatr Soc. 1998;46:210-5.

18. Weiner MF, Koss E, Wild KV, et al. Measures of psychiatric symptoms in Alzheimer patients: a review. Alzheimer Dis Assoc Disord. 1996;10:2030. Spring.

19. Wallihan DB, Stump TE, Callahan CM. Accuracy of self-reported health services use and patterns of care among urban older adults. Med Care. 1999;37:662-70.

20. Brandt JS, Folstein M. The telephone interview for cognitive status Neuropsychiatry Neuropsychol Behav Neurol. 1988;1(2):111-7.

21. Von Korff M, Wagner EH, Saunders K. A chronic disease score from automated pharmacy data. J Clin Epidemiol. 1992;45(2):197-203. Feb.

22. Kroenke K, Spitzer RL, Williams JB. The PHQ-9: validity of a brief depression severity measure. J Gen Intern Med. 2001;16(9):606-13. Sep.

23. Cohen-Mansfield $\mathbf{J}$. Nonpharmacologic interventions for inappropriate behaviors in dementia: a review, summary, and critique. Am J Geriatr Psychiatry. 2001;9:361-81.

24. Callahan CM, Hui SL, Nienaber NA, Musicl BS, Tierney WM. Longitudinal study of depression and health services use among elderly primary care patients. JAGS. 1994;42(8):833-8. Aug.

25. Callahan CM, Boustani M, Unverzagt FW, et al. Effectiveness of collaborative care for older adults with Alzheimer disease in primary care: a randomized controlled trial. JAMA. 2006;295(18):2148-57.

26. Belle SH, Burgio L, Burns R, et al. Enhancing the quality of life of dementia caregivers from different ethnic or racial groups. Ann Intern Med. 2006;145(10):727-38

27. Vickrey BG, Mittman BS, Connor KI, et al. The effect of a disease management intervention on quality and outcomes of dementia care: a randomized, controlled trial. Ann Intern Med. 2006;145:713-26. 